\title{
Distant Supernovae and Cosmic Deceleration *
}

\author{
Bruno Leibundgut and Jason Spyromilio \\ European Southern Observatory \\ Karl-Schwarzschild-Strasse 2 \\ D-85748 Garching \\ Germany
}

\begin{abstract}
Distant supernovae can now be detected routinely. To date 34 supernovae at $z>0.1$ have been discovered. Among them are 12 Type Ia supernovae confirmed spectroscopically and suited to measure the cosmic deceleration when appropriately employed as standard candles. However, peak magnitudes have been determined for only two objects so far and a determination of $q_{0}$ is not yet possible.

We describe the current status of the searches and possible pitfalls of the method which rests on few basic assumptions. The importance of sufficient information on the distant events is stressed and the observations of SN 1995K are used as an example of the detailed procedures employed in the analysis. Only spectroscopic classification and light curves in at least two filter bands provide the basis to use correction schemes for the luminosity which have successfully been established in nearby samples.

Time dilation has been detected acting on the light curve of SN $1995 \mathrm{~K}$ at a redshift of 0.478 , providing clear evidence of universal expansion. The observations are fully consistent with local Type Ia supernovae in an expanding universe but incompatible with the expectations from a static universe.

The contributions of the new, large telescopes to this research area are described. The extension of the observations to even more distant objects will provide a better leverage to distinguish between the possible decelerations and the inclusion of Type II supernovae into the sample add an independent check on the cosmological distances.
\end{abstract}

\section{Introduction}

Observational cosmology has made use of supernovae for several decades now (Zwicky 1965, Kowal 1968). Their large luminosity makes them suitable for detection at the distances required for the measurement of cosmological parameters. The main drawback of supernovae is the brief time they shine at this luminosity and that they are intrinsically very rare events.

In principle, all supernova types can be used for cosmological purposes. Explosions of massive stars, which are normally recognized by strong hydrogen lines in their spectrum (Type II supernovae), can be physically understood when the radiation hydrodynamics are solved (Eastman et al. 1996) and yield distances through the expanding photosphere method (Schmidt et al. 1992, 1994). The explosive carbon incineration of white dwarfs produces very luminous events with no hydrogen in their spectra - Type Ia supernovae (SNe Ia). These objects display a fairly uniform behavior which led to their use as standard candles

\footnotetext{
^ To appear in The Early Universe with the VLT, ed. J. Bergeron, (Berlin: Springer)
} 
(Kowal 1968, Tammann \& Leibundgut 1990, Branch \& Miller 1993, Sandage et al. 1996). The simple adoption of a unique peak luminosity, however, has recently been challenged by SN Ia samples with accurate relative distances and a dispersion of 0.6 magnitudes in maximum luminosity (Phillips 1993, Hamuy et al. 1995). The uniformity of the class is further eroded by correlations found in the light curve shapes and spectral appearances (Phillips 1993, Hamuy et al. 1995, Nugent et al. 1995, Vacca \& Leibundgut 1996). Local samples can be corrected by the proposed light curve shape fitting and only a small residual scatter (<0.2 mag.) remains (Hamuy et al. 1995, Riess et al. 1996).

Despite the variety of SNe Ia in the nearby universe we are still confident that the mechanisms are in place to use them at large distances for the determination of cosmological parameters, provided sufficient observations of each distant event are available.

The value of the Hubble constant has been the main focus of SN Ia applications (Sandage et al. 1996, Branch et al. 1996, Hamuy et al. 1995, Riess et al. 1996). The discrepancies in the reported results can be traced to the absolute nature of the measurement. The discussion of Riess et al. (1996) exemplifies the positions and assumptions adopted by several groups and resolves some of the conflicts rather convincingly.

Measuring the cosmic deceleration is fundamentally simpler, since it is done through relative measures. It is based on the comparison of the apparent brightness of objects with known relative luminosity at largely different redshifts. The ability to find the luminosity of a distant SN Ia relative to a nearby twin rests purely on observables (Schmidt et al. 1996, Perlmutter et al. 1996).

In this paper we present the basic ingredients necessary for successful detection and observation of distant supernovae and describe the current status of projects which use SNe Ia to measure $q_{0}(\S 2)$. In section 3 we develop the time dilation test for universal expansion as applied to SN 1995K. The prospects for and the contributions of the VLT to experiments like this one are presented together with the conclusions.

\section{Observing distant supernovae}

Finding distant $(z>0.3)$ supernovae is hampered by their variability and the rareness with which they occur. To overcome these obstacles a massive approach is needed. This has become possible with the development of sensitive, panoramic CCD detectors. Faint brightness levels have to be reached to increase the period a supernova is detectable. At the same time a large number of galaxies has to be surveyed to increase the chance of catching a supernova in the act.

There are a few pitfalls in the use of supernovae for accurate cosmological distance determinations. The first one - to find the objects - has been overcome by the concerted efforts at $4 \mathrm{~m}$ telescopes to image many galaxies in a short time period. Typically prime focus cameras with their large field of view are employed. The search fields, some including known clusters at $\mathrm{z}>0.3$, are observed after new moon to be re-imaged at the beginning of the next dark period. This ensures 
that all events detected during the second run are still on their rising branch and their light curve peak can be observed (Perlmutter et al. 1996). The currently employed strategy detects several supernovae (5-10) in such a search period (Garnavich et al. 1996a,b, Perlmutter et al. 1995b, 1996). All candidate objects for which it was possible to obtain a spectrum have been confirmed as supernovae at large distances.

Spectroscopic classification is of paramount importance as only Type Ia supernovae should be used for this method. Furthermore, on has to discriminate against SNe Ia which show clear peculiarities (SN 1991bg: Filippenko et al. 1992a, Leibundgut et al. 1993, Turatto et al. 1996; SN 1992K: Hamuy et al. 1994; SN 1991T: Filippenko et al. 1992b, Phillips et al. 1992, Ruiz-Lapuente et al. 1991; SN 1992bc: Maza et al. 1994) to avoid contamination of the sample. Since a $\mathrm{SN}$ Ia reaches about $\mathrm{R}=22.5$ at $\mathrm{z}=0.5$, the required spectroscopy stretches the capabilities of the current $4 \mathrm{~m}$ telescopes to the limit. The ability to schedule the spectroscopic follow-up observations ahead of time has proven to be essential for their success. Thanks to the combined efforts by several observatories has it been possible to obtain a spectrum for a fair fraction of the events (see below).

A further complication is the correction required to compare the observed flux evolution to the rest frame light curves of local supernovae. This K-correction, which depends on the phase of the supernova, can be overcome in two ways. One possibility is to use special filters corresponding to redshifted $\mathrm{B}$ and $\mathrm{V}$ passbands to minimize the corrections and also largely remove the dependence on phase. The other approach is to use standard broad-band filters which are closest to B and $\mathrm{V}$ in the rest frame of the supernova. Such K-corrections for regular SNe Ia have been calculated (Hamuy et al. 1993, Kim et al. 1996). In practice, this problem does not contribute significantly to the error budget.

Fig. 1. Comparison of the spectrum of SN 1995K with local SNe Ia near maximum light. The spectra are plotted in rest frame wavelengths.

Finally, photometry in two filters delivers the color of the supernova. The multi-color light curve shape method (Riess et al. 1996) makes use of this information to correct for possible absorption. Potentially absorption is one of the major contributors to the uncertainty in the derivation of $q_{0}$.

The first distant supernova was discovered in a dedicated search already in 1988. The object located in a galaxy cluster at $\mathrm{z}=0.31$ (SN 1988U, AC 118) was discovered about two weeks after maximum light and the spectrum suggested a Type Ia classification (Nørgaard-Nielsen et al. 1989). The same search reported a second event at a somewhat smaller redshift (SN 1988T, z=0.28; Hansen et al. 1989). The classification of this object is uncertain.

In recent years two major efforts to find and observe distant supernovae with the goal to determine the geometry of the universe have emerged. SN 1992bi was reported at a redshift of 0.458 by the Berkeley Cosmology Project and proved 
that it is possible to obtain an accurate light curve (Perlmutter et al. 1995a). The High-Z Supernova Team (Schmidt et al. 1995, 1996) has reported its observations of SN 1995K which include light curves in two filters and a spectrum near maximum light. The event occurred in a galaxy at a redshift of 0.478 and appears superposed on a spiral arm. The galaxy spectrum displays $\mathrm{H} \alpha, \mathrm{H} \beta$, [O II] and [O III] lines in emission and is consistent with an Sbc classification. The supernova spectrum itself had to be corrected for galaxy contamination and heavily smoothed. The result is a spectrum which displays the usual absorption and emission features of a Type Ia supernova near maximum light (Figure 1). All lines are shifted to the same redshift as determined for the galaxy confirming the association. A comparison of the SN 1995K spectrum with nearby events clearly shows the close relation to regular SNe Ia. The closest match is the near-maximum spectrum of SN 1989B. The comparison with the overluminous SN 1991T exemplifies the importance of spectroscopic classification. All spectral features of SN 1995K are stronger than in SN 1991T. In particular, the deep absorption due to Si II is characteristic of 'normal' SNe Ia (Nugent et al. 1995)

Fig. 2. B and V rest frame light curves of SN 1995K. The best $\chi^{2}$ fits for a simultaneous fit to the dilated (dark) and non-dilated (grey) light curves of SN 1991T are shown.

and distinguishes SN 1995K from SN 1991T. Similarly, the absorption ascribed to Ti II in low-temperature supernovae as observed in SN 1991bg near maximum is missing in SN 1995K. The phase of the spectrum is independently set by the light curve (see below) and is fully consistent with the spectral observation.

Light curves have been obtained in special $\mathrm{B}$ and $\mathrm{V}$ filters which correspond to a redshift of 0.45 and the Kron-Cousins $\mathrm{R}$ and I filters. At the redshift of SN $1995 \mathrm{~K}(\mathrm{z}=0.478)$ the transmission curve of the $\mathrm{R}$ filter is almost identical with the redshifted $\mathrm{B}$ passband and $\mathrm{K}$-corrections reduce to a constant describing the zero-point offset between the B and R passbands (see also Kim et al. 1996). We hence combined the B45 and R data sets as well as the V45 and I filter observations, respectively. The photometry covers the maximum phase quite adequately (Figure 2). The light curves span from about 18 days before until 35 days after maximum in B and from about peak light to 30 days thereafter in V. In fact, the first two measurements are from pre-discovery search observations. The supernova was discovered close to maximum light. The detection was not triggered in the earlier observations due to the faintness of the object $\left(\mathrm{R}_{\max } \approx 22.2\right)$ and its projection onto the high surface-brightness disc of the galaxy (e.g. Leibundgut et al. 1995). The pre-maximum points are essential for the determination of accurate light curve fits (Leibundgut et al. 1996) and a reliable measurement of the peak brightness.

As of June 199628 supernovae at redshifts larger than 0.3 have been reported. Of those at least 9 have been confirmed spectroscopically as being of Type Ia (Nørgaard-Nielsen et al. 1989, Schmidt et al. 1995, Perlmutter et al. 
Fig. 3. Histogram of distant supernovae reported before June 1996.

1995b, Garnavich et al. 1996a, b). Several supernovae without spectra have light curves typical of SNe Ia. The peak is covered in most cases, but modeling is still required for an accurate determination of the maximum brightness (cf. Perlmutter et al. 1996). The histogram of all supernovae discovered in these campaigns shows a clear concentration to the range of $0.3<z<0.5$ with $70 \%$ of all events detected so far (Figure 3). Monte Carlo simulations indicate that with the accuracy achieved for SN $1995 \mathrm{~K}$ it will take about 30 SNe Ia to decrease the uncertainty on $q_{0}$ to about 0.1 ( $1 \sigma$, Schmidt et al. 1996). The remaining error is mostly due to the uncertainty in the normalization by the local supernovae. The application of corrections like the multi-color light curve shape method (Riess et al. 1996) require the availability of at least two filter light curves and sufficient photometry coverage. Of course, it also implies that distant supernovae are indeed relatives of the nearby events and the training set employed to find the 'corrections' according to light curve shape and color is applicable.

Fig. 4. Hubble diagram of supernovae with the lines of constant luminosity in different mass universes. The horizontal bars show the redshifts of known distant supernovae. Spectroscopically classified SNe Ia are indicated by thick bars, SNe II and unclassified objects by short, thin lines.

The current status of the determination of $q_{0}$ is summarized in Figure 4 (cf. also Perlmutter et al. 1996). There are three SNe plotted at redshifts larger than 0.3. Two have spectroscopic SN Ia classifications (SN 1988U and SN 1995K) and the third (SN 1992bi) displays a light curve typical of SNe Ia. SN 1995K and SN 1992bi have been observed through their maximum and have fairly secure peak magnitudes, while SN $1988 \mathrm{U}$ has been extrapolated to maximum. Clearly it is too early to estimate the size of any cosmic deceleration with these three objects. Nevertheless, it is encouraging that the two distant objects fall between the lines for $1 / 2>q_{0}>0$. Note that we have used the normalization of the 'uncorrected' nearby sample of Hamuy et al. (1995). No correction for the light curve shape has been applied to the distant supernovae either. Light curve shape corrections change the zero-point of the normalization and have to be applied carefully. This further exemplifies the importance of a large knowledge base for the distant supernovae to confirm their conformity with the local samples.

The redshifts of additional supernovae already discovered in the two projects are indicated in Fig. 4. It appears that quite a few events have been recorded to date and that we should be able to find the value for the deceleration fairly soon. However, not all objects are indeed SNe Ia. Some have no spectroscopic confirmation while others are known to be Type II. The importance of sufficient light curve coverage is another limiting factor. Figure 4 thus presents a very 
optimistic view and we will not be able to fill the diagram with a point for each line indicating a SN redshift. In the next year a similar amount of distant events will be discovered and soon we should be able to measure $q_{0}$.

\section{Time Dilation}

A significant result, which can be derived from the single event currently available to us, is the direct observation of time dilation due to universal expansion. The light curve of a distant supernova acts as a clock, the ticks of which can be directly compared to wavefront stretching as observed in the spectra. In an expanding universe the two have to go together while other theories of redshift invoke energy dissipation of photons or similar mechanisms (Arp 1987, Arp et al. 1990). Two distant SNe Ia have been observed at least 18 days before maximum (SN 1994am, Perlmutter et al. 1996, and SN 1995K). No local event has been observed at such an early phase. The earliest observations of nearby SNe Ia are reported in the range of 14 days before peak (e.g. SN 1990N; Leibundgut et al. 1991). Moreover, the brightness evolution of SN $1995 \mathrm{~K}$ is the slowest ever observed for any SN Ia (Leibundgut et al. 1996). In Figure 2 we have overplotted the best fits as determined by minimization of $\chi^{2}$ to the combined $\mathrm{B}$ and $\mathrm{V}$ rest frame dilated and non-dilated light curves. For the comparison we have chosen one of the slowest local supernovae known, SN 1991T. The non-dilated curves cannot fit the observations, while the photometry of SN 1995K is fully consistent with the dilated template. Analyses with comparison light curves of other local events confirm this result (Leibundgut et al. 1996). The combination of regular color, typical spectrum, a luminosity in the range of reasonable deceleration parameter values, and the conforming light curve of SN 1995K strongly favor the dilated case. In a non-expanding universe SN 1995K would represent a weird event displaying a regular spectrum but deviating strongly from the locally established luminosity-decline rate relation, and displaying the slowest decline of all known SNe Ia. Malmquist bias could not explain this supernova as it would be less luminous than local SNe Ia in a static universe. The same conclusions was found based on light curves in a single filter for a set of seven distant SNe $(0.3<z<0.5)$ three of which with a spectroscopic Ia classification (Goldhaber et al. 1996).

\section{Conclusions}

Finding supernovae at redshifts above 0.3 has become a routine enterprise over the last two years. There are currently two groups vigorously following this route to find the value of the deceleration parameter (Perlmutter et al. 1995a, 1996, Schmidt et al. 1996, Leibundgut et al. 1996). Several supernovae are found each semester in the scheduled search runs. However, the currently available data set is not quite large enough to make a serious attempt to measure $q_{0}$. Only a dozen objects has the required spectral classification and sufficient light curve coverage 
to provide the basis for an accurate measurement of the peak brightness and the correction to the luminosity from the light curve shape. There also remains too much slack in the zero-point of the expansion lines in the Hubble diagram as defined by local supernovae. This uncertainty directly goes into the estimate of the cosmic deceleration.

Another error source which has to be carefully excluded are systematic differences of distant events from the nearby ones. Thus the sample has to be tightly checked against possible evolutionary effects. The theoretical understanding of SNe Ia is not sound enough to exclude evolutionary effects which could change the luminosities of the explosions (Canal et al. 1996). Not all models predict such changes but some do. By a careful analysis of the distant sample we should be able to distinguish among the various current candidate models of SN Ia explosions.

Sufficient statistics and, eventually, detailed spectroscopic follow-up observations should be able to detect such differences.

The large aperture telescopes will provide the necessary spectroscopy for the classification of the supernovae. Their rôle will be in the detection and follow up observations of more distant events. SNe Ia at maximum should be around $\mathrm{I} \approx 25$ at $\mathrm{z}=1$ and the difference between an empty and a critical universe is 0.54 magnitudes providing more leverage for the determination of $q_{0}$. The complication of a cosmological constant can also be explored with standard candles at large distances (Goobar \& Perlmutter 1995). The infrared capabilities of the large telescopes are of paramount importance as the rest frame $\mathrm{V}$ light is shifted into the $\mathrm{J}$ band at $\mathrm{z}=1$.

Important cosmological tests also remain for the VLT and its 8m-class partners. The time dilation as observed in the spectral evolution of a distant SN Ia would provide an even more stringent proof of cosmic expansion. The distances derived for Type II supernovae through the expanding photosphere method (Eastman et al. 1996) are based on the combination of a luminosity distance with an angular diameter distance. These two distances are related in all cosmological models (Carroll, Press, \& Turner 1992). Since these explosions are typically less luminous than SNe Ia only the large telescopes will be able to deliver the signal needed to measure the expansion velocities accurately.

Acknowledgment: It is a pleasure to thank our collaborators in this experiment. Some of the data presented in this review has been kindly provided before publication.

\section{References}

Arp, H. C. 1987, Quasars, Redshifts and Controversies, Interstellar Media, Berkeley

Arp, H. C., Burbidge, G. R., Hoyle, F., Narlikar, J. V., \& Wickramasinghe, N. C. 1990, Nature, 346, 807

Branch, D., \& Miller, D. L. 1993, ApJ, 405, L5

Branch, D., Romanishin, W., \& Baron, E. 1996, ApJ, 465, 73

Canal, R., Ruiz-Lapuente, P., \& Burkert, A. 1996, ApJ, 456, L101

Carroll, S. M., Press, W. H., \& Turner, E. L. 1992, ARA\&A, 30, 499 
Eastman, R. G., Schmidt, B. P., \& Kirshner, R. P. 1996, ApJ, in press

Filippenko, A. V., et al. 1992a, AJ, 104, 1534

Filippenko, A. V., et al. 1992b, ApJ, 384, L15

Garnavich, P., et al. 1996a, IAU Circ. 6332

Garnavich, P., et al. 1996b, IAU Circ. 6358

Goldhaber, G., et al. 1996, Thermonuclear Supernovae, eds. R. Canal, P. RuizLapuente, \& J. Isern, (Dordrecht: Kluwer), in press

Goobar, A \& Perlmutter, S. 1995, ApJ, 450, 14

Hamuy, M., Phillips, M. M., Wells, L. A., \& J. Maza 1993, PASP, 105, 787

Hamuy, M., Phillips, M. M., Maza, J., Suntzeff, N. B., Schommer, R. A., \& Avilés, R. 1995, AJ, 109, 1

Hamuy, M., et al. 1994, AJ, 108, 2226

Hansen, L., Jørgensen, H. E., Nørgaard-Nielsen, H. U., Ellis, R. S., \& Couch, W. J. 1989, A\&A, 211, L9

Kowal, C. T. 1968, AJ, 73, 1021

Leibundgut, B., Kirshner, R. P., Filippenko, A. V., Shields, J. C., Foltz, C. B., Phillips, M. M., \& Sonneborn, G. 1991, ApJ, 371, L23

Leibundgut, B., et al. 1993, AJ, 105, 301

Leibundgut, B., et al. 1995, The Messenger, 19

Leibundgut, B., et al. 1996, ApJ, 466, L21

Maza, J., Hamuy, M., Phillips, M. M., Suntzeff, N. B., Avilés, R. 1994, ApJ, 424, L107

Nørgaard-Nielsen, H. U., Hansen, L., Jørgensen, H. E., Salamanca, A. A., Ellis, R. S., \& Couch, W. J. 1989, Nature, 339, 523

Nugent, P., Phillips, M. M., Baron, E., Branch, D., \& Hauschildt, P. 1995, ApJ, 455, L147

Perlmutter, S., et al. 1995a, ApJ, 440, L41

Perlmutter, S., et al. 1995b, IAU Circ. 6270

Perlmutter, S., et al. 1996, Thermonuclear Supernovae, eds. R. Canal, P. RuizLapuente, \& J. Isern, (Dordrecht: Kluwer), in press

Phillips, M. M. 1993, ApJ, 413, L105

Phillips, M. M., Wells, L. A., Suntzeff, N. B., Hamuy, M., Leibundgut, B., Kirshner, R. P., \& Foltz, C. B. 1992, AJ, 103, 1632

Riess, A. G., Press, W. M., \& Kirshner, R. P. 1996, ApJ, in press

Ruiz-Lapuente, P., Cappellaro, E., Turatto, M., Gouiffes, C., Danziger, I. J., Della Valle, M., \& Lucy, L. B. 1992, ApJ, 387, L33

Sandage, A., Saha, A., Tammann, G. A., Labhardt, L., Panagia, N., \& Macchetto, F. D. 1996, ApJ, 460, L15

Schmidt, B. P., Kirshner, R. P., \& Eastman, R. G. 1992, ApJ, 395, 366

Schmidt, B. P., Kirshner, R. P., Eastman, R. G., Phillips, M. M., Suntzeff, N. B., Hamuy, M., Maza, J., \& Avilés, R. 1994, ApJ, 432, 4

Schmidt, B. P., et al. 1995, IAU Circ. 6160

Schmidt, B. P., et al. 1996, in preparation

Tammann, G. A., \& Leibundgut, B. 1990, A\&A, 236, 9

Turatto, M., Benetti, S., Cappellaro, E., Danziger, I. J., Della Valle, M., Gouiffes, C., Mazzali, P. A., \& Patat, F. 1996, MNRAS, in press

Vacca, W. D., \& Leibundgut, B. 1996, Thermonuclear Supernovae, eds. R. Canal, P. Ruiz-Lapuente, J. Isern, (Dordrecht: Kluwer), in press

Zwicky, F. 1965, Stellar Structure, eds. L. H. Aller \& D. B. McLaughlin, (Chicago: University of Chicago Press), 367 


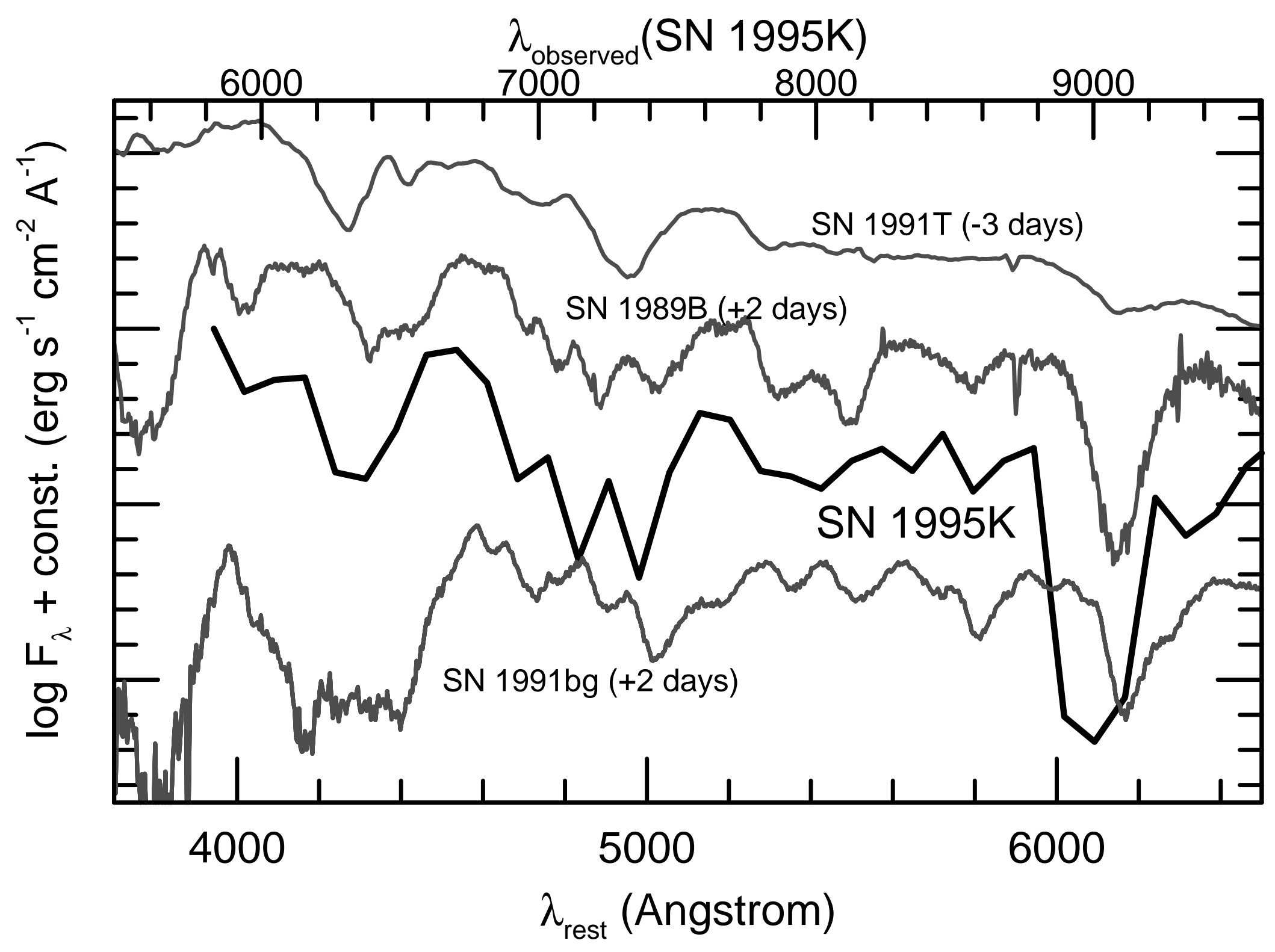




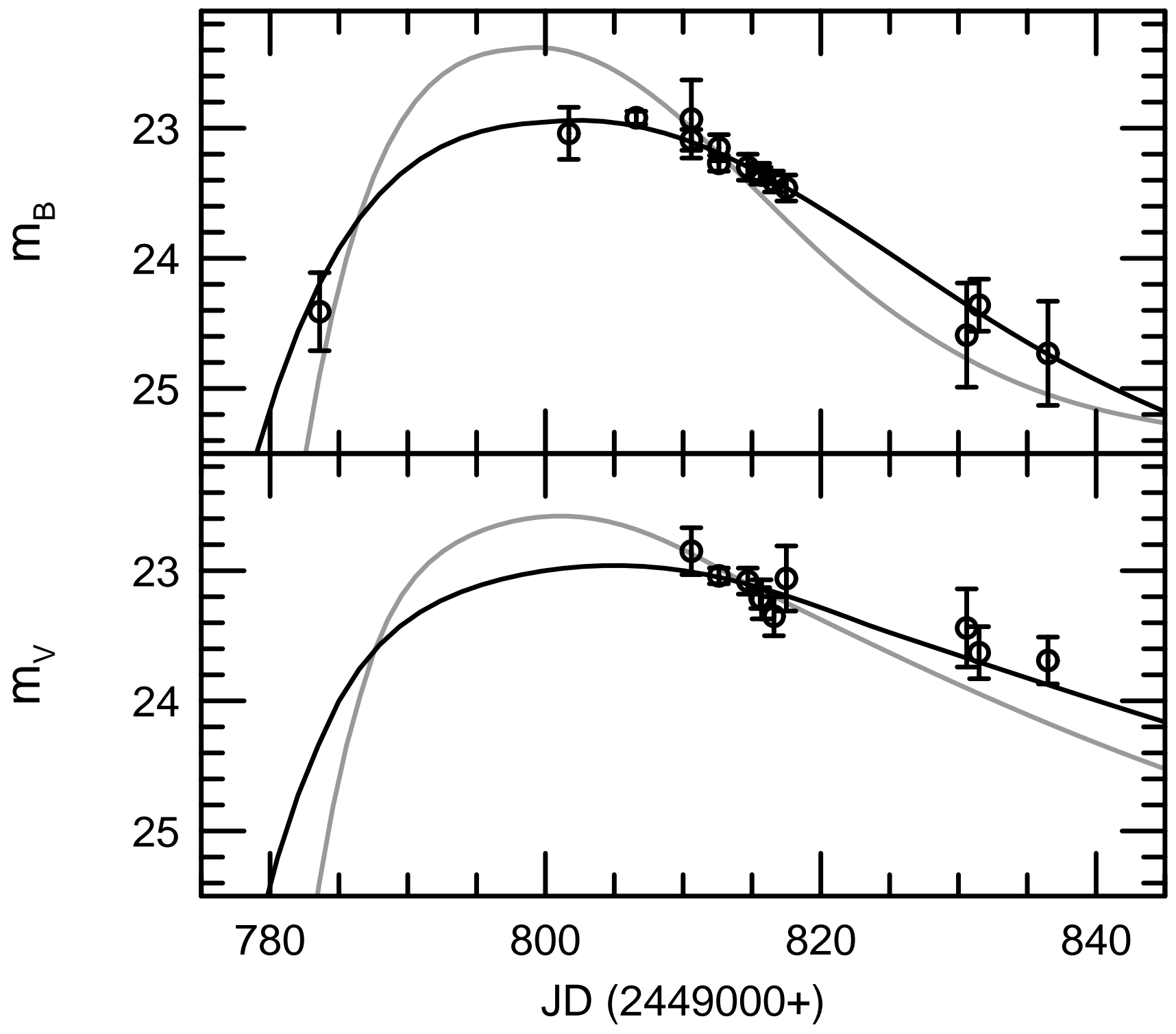




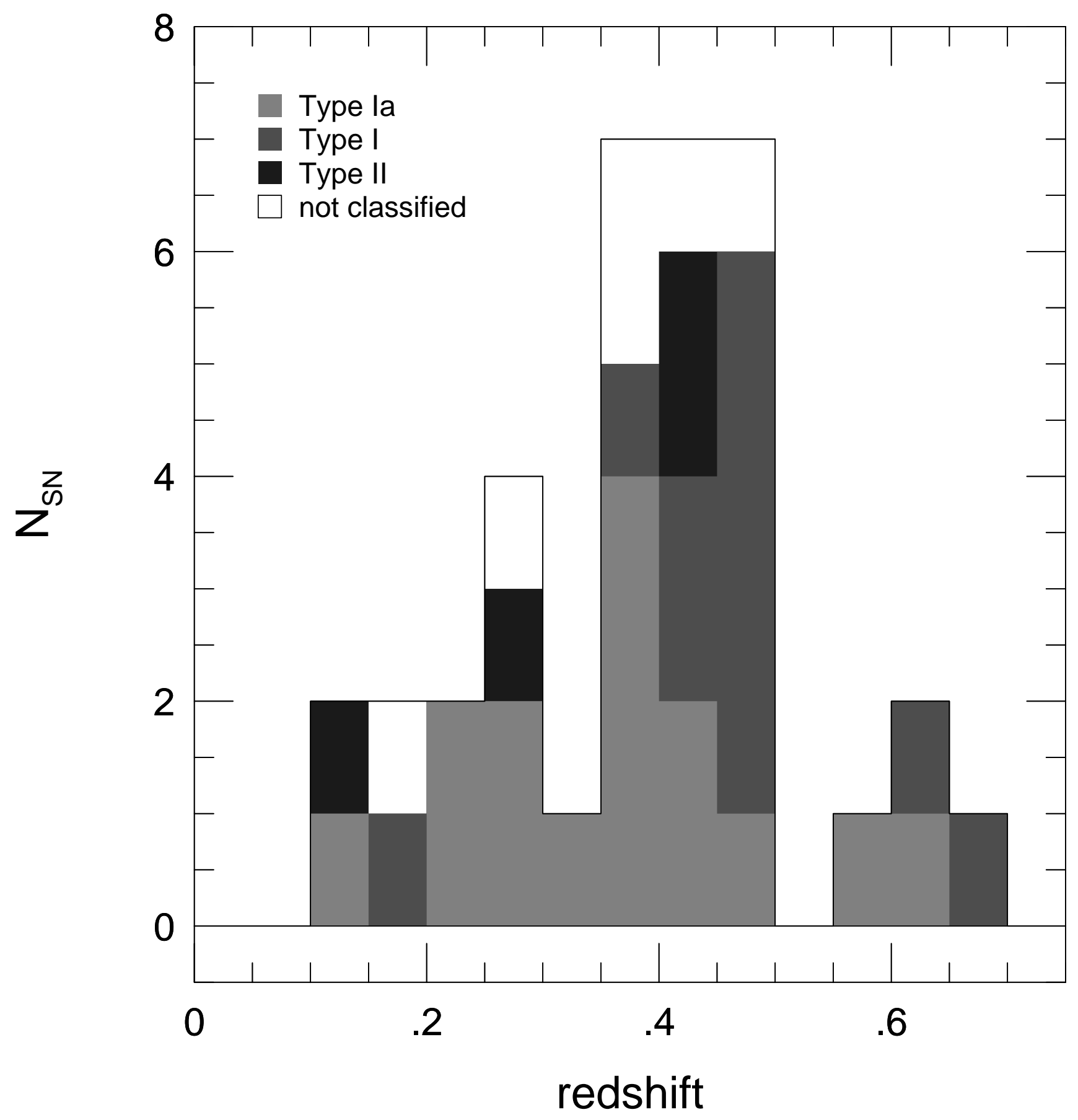




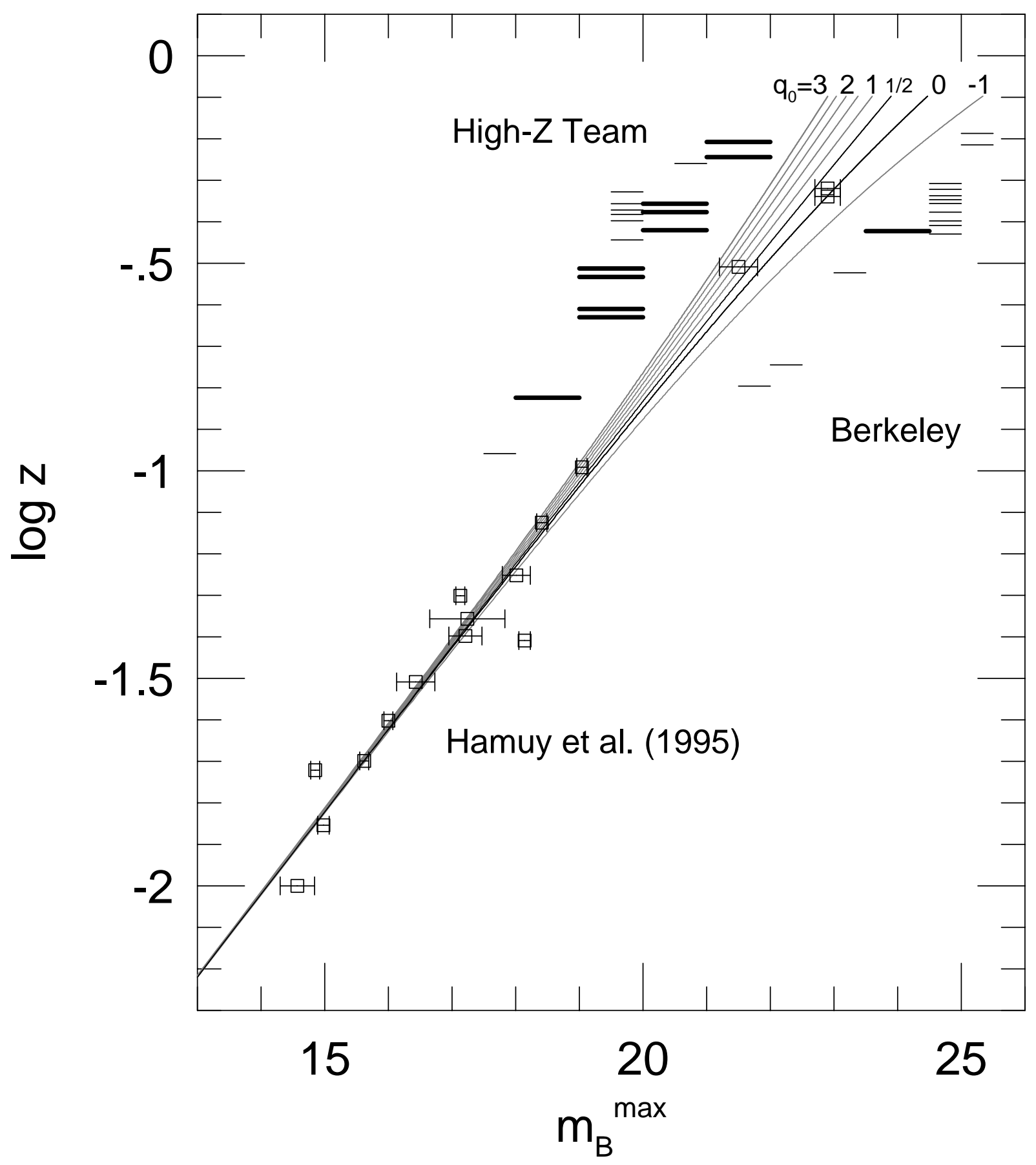

